\title{
Functional role and molecular mechanisms underlying prohibitin 2 in platelet mitophagy and activation
}

\author{
LONG-LONG HU $^{1 *}$, KAI ZOU ${ }^{1 *}$, YUAN CHEN ${ }^{1}$, LI-JUAN WU $^{1}$, JIE CAO $^{1}$, XIAO-YING XIONG ${ }^{1}$, \\ LING WANG $^{2}$, XIAO-SHU CHENG ${ }^{1}$, QING-ZHONG XIAO ${ }^{3}$ and REN-QIANG YANG ${ }^{1}$
}

\author{
${ }^{1}$ Department of Cardiology and ${ }^{2}$ Medicine Lab, The Second Affiliated Hospital of Nanchang University, Nanchang, \\ Jiangxi 330006, P.R. China; ${ }^{3}$ Department of Clinical Pharmacology, William Harvey Research Institute, Barts and \\ The London School of Medicine and Dentistry, Queen Mary University of London, London EC1M 6BQ, UK
}

Received August 17, 2020; Accepted February 24, 2021

DOI: $10.3892 / \mathrm{mmr} .2021 .12023$

\begin{abstract}
Platelet mitophagy is a major pathway involved in the clearance of injured mitochondria during hemostasis and thrombosis. Prohibitin 2 (PHB2) has recently emerged as an inner mitochondrial membrane receptor involved in mitophagy. However, the mechanisms underlying PHB2-mediated platelet mitophagy and activation are not completely understood. PHB2 is a highly conserved inner mitochondrial membrane protein that regulates mitochondrial assembly and function due to its unique localization on the mitochondrial membrane. The present study aimed to investigate the role and mechanism underlying PHB2 in platelet mitophagy and activation. Phorbol-12-myristate-13-acetate (PMA) was used to induce MEG-01 cells maturation and differentiate into platelets following PHB2 knockdown. Cell Counting Kit-8 assays were performed to examine platelet viability. Flow cytometry was performed to assess platelet mitochondrial membrane potential. RT-qPCR and western blotting were conducted to measure mRNA and protein expression levels, respectively. Subsequently, platelets were exposed to CCCP and the role of PHB2 was assessed. The results of the present study identified a crucial role for PHB2 in platelet mitophagy and activation, suggesting that PHB2-mediated regulation of mitophagy may serve as a novel strategy for downregulating the expression of platelet activation genes. Although further research into
\end{abstract}

Correspondence to: Professor Ren-Qiang Yang, Department of Cardiology, The Second Affiliated Hospital of Nanchang University, 1 Minde Road, Nanchang, Jiangxi 330006, P.R. China

E-mail: yangrenqiangcn@163.com

Professor Qing-Zhong Xiao, Department of Clinical Pharmacology, William Harvey Research Institute, Barts and The London School of Medicine and Dentistry, Queen Mary University of London, 101 Charterhouse Square, London EC1M 6BQ, UK

E-mail: q.xiao@qmul.ac.uk

*Contributed equally

Key words: platelet activation, mitophagy, prohibitin 2 mitophagy is required, the present study suggested that PHB2 may serve as a novel therapeutic target for thrombosis-related diseases due to its unique localization on the mitochondrial membrane.

\section{Introduction}

Prohibitin (PHB) is ubiquitously expressed in cells and is primarily localized on the inner mitochondrial membrane. PHB is an evolutionarily, highly conserved protein that serves as a molecular chaperone in the assembly of the mitochondrial respiratory chain complex subunit $(1,2)$. PHB often exists in the form of a complex, consisting of 12-16 heterogenous dimer pairs of PHB1 and PHB2, which are closely related, that can be anchored to the mitochondrial intima to form a ring-shaped barrier structure $(3,4)$.

The location of PHB2 proteins determines its function. For example, the presence of PHB2 on the inner mitochondrial membrane aids with maintaining the normal morphology of the mitochondria, as well as anti-oxidative stress and apoptosis levels, thereby affecting mitochondrial function (5). A previous study reported that hyperglycemia in patients with type 2 diabetes promoted ATP production and decreased the mitochondrial membrane potential $(\Delta \Psi m)$ in platelet mitochondria (6). In addition, another previous study reported that platelet mitochondrial activity was transiently enhanced, mitochondrial function was disordered, and the production of ATP and reactive oxygen species was increased in patients with diabetes (7).

Platelet activation is closely associated with mitochondrial dysfunction (8). A previous study demonstrated that cells primarily participated in mitochondrial renewal via the selective autophagy pathway (9). The process of selectively removing mitochondria by autophagy is known as mitophagy (10). Mitophagy maintains a balance in the number and quality of mitochondria in the cell by scavenging a portion of the damaged mitochondria. Furthermore, mitophagy not only removes damaged mitochondria, but also degrades healthy mitochondria. For example, the exposure of cells to a harsh environment promotes the synthesis of excess mitochondria to increase the operational burden of cells, which results in the degradation of healthy mitochondria to 
maintain cell survival (11). The process of autophagy has been reported to occur in platelets of humans and dogs, wherein inhibition of the autophagic signaling pathway or autophagic degradation prevents platelet aggregation and adhesion functions (12-14). Therefore, platelet autophagy was suggested to serve an important role in regulating platelet activation. A previous study demonstrated that mitophagy was induced in the platelets of patients with diabetes, which was considered to serve as an intrinsic mechanism that protected platelets from severe oxidative stress, in addition to preventing apoptosis and thrombosis (15). Therefore, the present study aimed to investigate the role and mechanism of action underlying PHB2 in platelet mitophagy and activation.

\section{Materials and methods}

MEG-01 cell culture. MEG-01 cells (American Type Culture Collection) were cultured in RPMI-1640 (Gibco; Thermo Fisher Scientific, Inc.) supplemented with $10 \%$ FBS (Gibco; Thermo Fisher Scientific, Inc.) at $37^{\circ} \mathrm{C}$ with $5 \% \mathrm{CO}_{2}$. For differentiation into platelets, MEG-01 cells were treated with $15 \mathrm{ng} / \mathrm{ml}$ phorbol-12-myristate-13-acetate (PMA) for 72 h. To observe cell morphology, air-dried cell smears were fixed using $0.2 \%$ Wright's staining solution for 2-3 sec at room temperature, followed by staining with Wright-Giemsa stain (Sigma-Aldrich; Merck KGaA) for 15-25 min at room temperature according to manufacturer's protocol. Stained cells were visualized using a light microscope (Olympus Corporation). PE-conjugated anti-CD61 (cat. no. 555754; BD Biosciences) was used to assess platelet activation via flow cytometry. Briefly, platelets were fixed with $4 \%$ paraformaldehyde at room temperature for $15 \mathrm{~min}$, rinsed with PBS and stained with anti-CD61 in the dark for 15-30 min. Subsequently, 1x10 4 total platelets per sample were acquired and analyzed using a FACSCalibur $^{\mathrm{TM}}$ flow cytometer (BD Biosciences) and FlowJo software (version 10; FlowJo LLC). CD61 mRNA expression levels were analyzed via reverse transcription-quantitative PCR (RT-qPCR).

Cell Counting Kit-8 (CCK-8) assay. Following PMA-induced differentiation of MEG-01 cells, platelets were uniformly seeded (100 $\mu \mathrm{l}$ cell suspension; $2 \times 10^{3}$ cells/well) into 96-well plates and treated with 0 (negative control; NC), 10, 20, 50, 70 or $100 \mu \mathrm{M}$ carbonyl cyanide 3-chlorophenylhydrazone (CCCP; cat. no. ab141229; Abcam), a mitochondrial oxidative phosphorylation uncoupler $(16,17)$. CCCP was diluted using DMSO (Sigma-Aldrich; Merck KGaA). A DMSO group was used in subsequent experiments to exclude the effects of DMSO on cells. For each treatment group, 10 replicate wells were used. Following incubation for $3 \mathrm{~h}$ at $37^{\circ} \mathrm{C}, 10 \mu \mathrm{l} \mathrm{CCK}-8$ solution (Dojindo Molecular Technologies, Inc.) was added to each well and incubated for $1 \mathrm{~h}$. In the blank control group, cell-free cell culture medium was added to the well, followed by CCK-8 solution according to the aforementioned protocol. Absorbance was measured at a wavelength of $450 \mathrm{~nm}$ using a microplate reader to obtain optical density values. Cell viability was calculated according to the following formula: Cell viability $(\%)=[($ experimental well-blank well $) /(\mathrm{NC}$ well-blank well)] x100.
Measurement of $\Delta \Psi_{m}$ via flow cytometry. Platelets $\left(5 \times 10^{6}\right)$ were obtained and resuspended in $0.5 \mathrm{ml}$ RPMI-1640 supplemented with $10 \%$ FBS. Subsequently, $0.5 \mathrm{ml}$ prepared JC-1 staining working solution (BD Biosciences) was added to the cell suspension, gently mixed by pipetting and incubated at $37^{\circ} \mathrm{C}$ for $20 \mathrm{~min}$. Subsequently, the suspension was centrifuged at $4^{\circ} \mathrm{C}$ at $600 \mathrm{x}$ g for $5 \mathrm{~min}$ and the supernatant was discarded. The precipitate was washed with JC-1 staining buffer (1X) as follows: Cells were resuspended in $1 \mathrm{ml}$ JC-1 staining buffer (1X) and centrifuged at $600 \mathrm{x}$ g at $4^{\circ} \mathrm{C}$ for $5 \mathrm{~min}$; the supernatant was discarded; and the washing procedure was repeated three times. Subsequently, cells were resuspended in $500 \mu \mathrm{l} \mathrm{JC-1}$ Binding Buffer (1X) and analyzed using a FACSCalibur ${ }^{\mathrm{TM}}$ flow cytometer (BD Biosciences). Compensation for FL-1/FL-2 was set up using FACSComp ${ }^{\mathrm{TM}}$ beads (BD Biosciences) and FlowJo software (version 10; FlowJo LLC) was used to set up the flow cytometer.

$R T-q P C R$. Total RNA was extracted from MEG-01 cells using TRIzol ${ }^{\circledR}$ reagent (Invitrogen; Thermo Fisher Scientific, Inc.). Total RNA was reverse transcribed into cDNA using random hexamer primers and a TaqMan reverse transcription kit (Takara Bio, Inc.) according to the manufacturer's protocol. The temperature protocol used for RT was $42^{\circ} \mathrm{C}$ for $2 \mathrm{~min}, 37^{\circ} \mathrm{C}$ for $15 \mathrm{~min}$ and $85^{\circ} \mathrm{C}$ for $5 \mathrm{sec}$. Subsequently, qPCR was performed using a SYBR Premix Ex Taq II kit (Takara Bio, Inc.) on a 7900HT Fast Real-Time PCR system (Applied Biosystems; Thermo Fisher Scientific, Inc.). The following thermocycling conditions were used for qPCR: Pre-denaturation at $95^{\circ} \mathrm{C}$ for $30 \mathrm{sec}$; followed by 40 amplification cycles (denaturation at $95^{\circ} \mathrm{C}$ for $5 \mathrm{sec}$, annealing at $60^{\circ} \mathrm{C}$ for $34 \mathrm{sec}$ and extension at $95^{\circ} \mathrm{C}$ for $15 \mathrm{sec}$ ). The sequences of the primers used for qPCR are listed in Table I. mRNA expression levels were quantified using the $2^{-\Delta \Delta \mathrm{Cq}}$ method (18) and normalized to the internal reference gene GAPDH. the reference gene.

Western blotting. Total proteins were extracted from platelets using RIPA lysis buffer (Cell Signaling Technology, Inc.) according to the manufacturer's protocol. Protein concentrations were quantified using a bicinchoninic acid protein assay kit (Thermo Fisher Scientific, Inc.). Subsequently, proteins $(30 \mu \mathrm{g})$ were separated via $12.5 \%$ SDS-PAGE and electrotransferred onto PVDF membranes. Following blocking with 3\% (w/v) BSA (Sigma-Aldrich; Merck KGaA) in TBST $[150 \mathrm{mM} \mathrm{NaCl}, 10 \mathrm{mM}$ Tris- $\mathrm{HCl}(\mathrm{pH} 7.5)$ and $0.1 \%$ Tween-20] at room temperature for $1 \mathrm{~h}$, the membranes were incubated overnight at $4^{\circ} \mathrm{C}$ with the following primary antibodies: Anti-PHB2 (1:1,000; cat. no. sc-133094; Santa Cruz Biotechnology, Inc.), anti-LC3B (1:2,000; cat. no. ab48394; Abcam) and anti- $\beta$-tubulin $(1: 5,000$; cat. no. 10068-1-AP; ProteinTech Group, Inc.). Following the primary antibody incubation, the membranes were incubated with HRP-conjugated goat anti-mouse IgG (1:2,000; cat. no. SA00001-1; ProteinTech Group, Inc.) and goat anti-rabbit IgG (1:2,000; cat. no. SA00001-2; ProteinTech Group, Inc.) secondary antibodies for $2 \mathrm{~h}$ at room temperature. Protein bands were visualized using Pierce ${ }^{\mathrm{TM}}$ ECL Western Blotting Substrate (cat. no. 32109; Thermo Fisher Scientific, Inc.) on a ChemiDoc XRS imager (Bio-Rad Laboratories, Inc.). Protein expression levels were semi-quantified using 
Table I. Sequences of primers used for quantitative PCR.

\begin{tabular}{ll}
\hline Gene & \multicolumn{1}{c}{ Sequence $\left(5^{\prime} \rightarrow 3^{\prime}\right)$} \\
\hline Prohibitin 2 & F: ACACTCGTTCCTCGTAGTCC \\
& R: TTGGTTCCAGTACCCCATTA \\
CD61 & F: TTAGGTTCAGCTTGGGCCTG \\
& R: TTGGAGACACGGTGAGCTTC \\
GAPDH & F: GATTTTGGAGGGATCTCGCT \\
& R: CAGGGCTGCTTTTACTCTGGT
\end{tabular}

F, forward; R, reverse.

Image Lab software (version 4.0.1; Bio-Rad Laboratories, Inc.) with $\beta$-tubulin as the loading control.

Cell transfection. Short hairpin RNAs (shRNAs/shs) targeting PHB2 were designed and synthesized by Shanghai GeneChem Co. Ltd. The recombinant lentivirus (LV) of shRNA targeting PHB2 [PHB2-RNA interference (RNAi)-LV] and control LV (GFP LV) were commercially prepared by Shanghai GeneChem Co. Ltd. Briefly, a LV transfer vector (GV248) was constructed, which contained a puromycin resistance gene and an enhanced GFP gene. shRNA expression was driven by a U6 promoter. To package viruses, a 2nd generation system was used and 293T cells (The Cell Bank of Type Culture Collection of The Chinese Academy of Sciences).were transiently transfected with $20 \mu \mathrm{g}$ GV248 transfer plasmid and three packaging vectors: i) $20 \mu \mathrm{g}$ pGC-LV; ii) $15 \mu \mathrm{g}$ pHelper 1.0 ; and iii) $10 \mu \mathrm{g}$ pHelper 2.0 (all Shanghai GeneChem Co., Ltd.). Following transfection for $72 \mathrm{~h}$, lentiviral particles were collected, filtered and concentrated by ultracentrifugation at 12,000 x g for $2 \mathrm{~h}$ at $4^{\circ} \mathrm{C}$. At $30-50 \%$ confluence, MEG-01 cells were transduced with lentiviral shRNA (100 plaque-forming unit/cell; MOI, 100). At $48 \mathrm{~h}$ post-transduction, transduction efficiencies were evaluated via RT-qPCR. At $72 \mathrm{~h}$ post-transduction, PHB2 protein expression levels were analyzed via western blotting. The targeting sequences of the shRNAs were as follows: LV-PHB2-RNAi (57874-1), 5'-AGAATATCTCCAAGACGA T-3'; LV-PHB2-RNAi (57875-1), 5'-TGAGCTTTAGCCGAG AGTA3'; LV-PHB2-RNAi (57876-2), 5'-TAGCATGTACCA GCGCCTA-3'; and NC-RNAi (CON077), 5'-TTCTCCGAA CGTGTCACGT-3'.

mRNA sequencing ( $m R N A$-seq). mRNA-seq experiments were performed by Novogene Co., Ltd. An mRNA-seq library was prepared for sequencing using standard Illumina protocols (19). Briefly, total RNA was extracted from platelets with or without transfection of shPHB2 treatment using TRIzol reagent (cat. no. 15596026, Invitrogen; Thermo Fisher Scientific, Inc.). To remove any contaminating genomic DNA, total RNA was treated with RNase-free DNase I (cat. no. M0303L; New England BioLabs, Inc.). RIN value (RNA integrity number) was used as the criterion to determine the integrity of the sample RNA using the RNA Nano 6000 Assay Kit (cat. no. 5067-1511; Agilent Technologies, Inc.) and the Bioanalyzer 2100 System (Agilent Technologies, Inc.). mRNA extraction was performed using Dynabeads oligo(dT) (cat. no. 61002; Invitrogen; Thermo Fisher Scientific, Inc.). Double-stranded cDNA was synthesized using Superscript II reverse transcriptase (cat. no. 18-064-022; Invitrogen; Thermo Fisher Scientific, Inc.) and random hexamer primers. Subsequently, cDNAs were fragmented by nebulization and the standard Illumina protocol was followed to generate the mRNA-seq library using the NEBNext ${ }^{\circledR}$ Ultra $^{\text {TM }}$ RNA Library Prep Kit for Illumina ${ }^{\circledR}$ (cat. no. E7530L; New England Biolabs, Inc.). The sequencing type we used was PE150 (Hiseq X; Illumina, Inc.). The inserted fragment length was 250-300 bp and the sequencing direction was $5^{\prime} \rightarrow 3^{\prime}$. After the library was constructed, a Qubit 2.0 Fluorometer (Invitrogen; Thermo Fisher Scientific, Inc.) was used for preliminary quantification, followed by dilution and detection using $1.5 \mathrm{ng} / \mu \mathrm{l}$ sample and a CFX96 Touch Real-Time PCR Detection System (Bio-Rad Laboratories, Inc.). For data analysis, basecalls were performed using cassava. Reads were aligned to the genome with the split read aligner TopHat (version 2.0.7, http://tophat.cbcb.umd.edu) and Bowtie2 (version 2.4.2; http://bowtie-bio.sourceforge.net), using default parameters. HTSeq (version 0.6.0, https://htseq.readthedocs.io) was used to estimate the abundance of the reads.

Gene Ontology (GO) functional term and Kyoto Encyclopedia of Genes and Genomes (KEGG) signaling pathway enrichment analyses. GO functional term enrichment analysis of differentially expressed genes (DEGs; $\mid \log 2$ (FoldChange) $\mid>1$ and adjusted $\mathrm{P}$-value<0.05) was conducted using the GOseq $\mathrm{R}$ package (20). Briefly, GO analysis was performed by collating the significantly enriched GO terms in the identified DEGs. Subsequently, DEGs were filtered based on biological functions and mapped to GO terms in the GO database (www. geneontology.org). Gene numbers were calculated for each term using the hypergeometric test to obtain significantly enriched GO terms for DEGs, which were compared with sh-NC. GO terms with a corrected $\mathrm{P}<0.05$ were considered as significantly enriched in DEGs. KEGG Orthology Based Annotation System software (version no. 4.0.1; bioconductor. org/packages/release/bioc/html/clusterProfiler.html) was used to perform KEGG pathway enrichment analysis and to determine the statistical enrichment of the DEGs in KEGG signaling pathways (www.genome.jp/kegg) $(21,22)$. The analysis was used to identify the significant enrichment of genes involved in metabolic or signaling pathways.

Statistical analysis. Statistical analyses were performed using GraphPad Prism software (version 7.0; GraphPad Software, Inc.). Data are presented as the mean \pm SD. Comparisons between two groups were analyzed using an unpaired Student's t-test, whereas comparisons among multiple groups were analyzed using one-way ANOVA followed by Bonferroni's post hoc test. $\mathrm{P}<0.05$ was considered to indicate a statistically significant difference. All experiments were repeated three times.

\section{Results}

MEG-01 cells are successfully induced to mature and differentiate into platelets using PMA in vitro. MEG-01 cells are megakaryocyte leukemia cells, which were cultured 

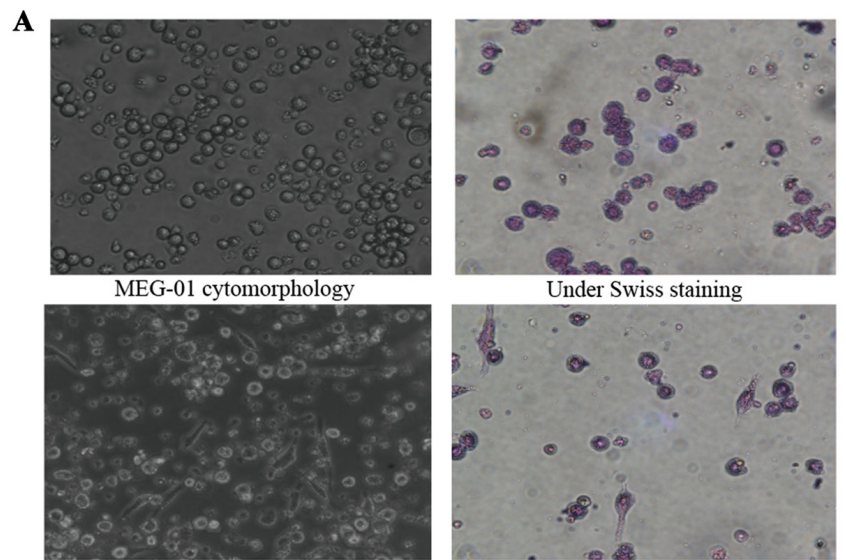

After PMA induction for $72 \mathrm{~h}$

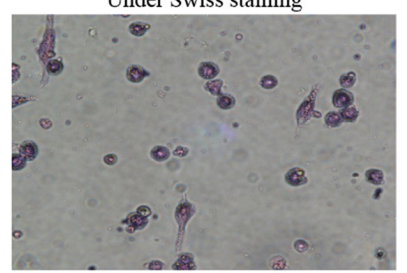

Under Swiss staining

B
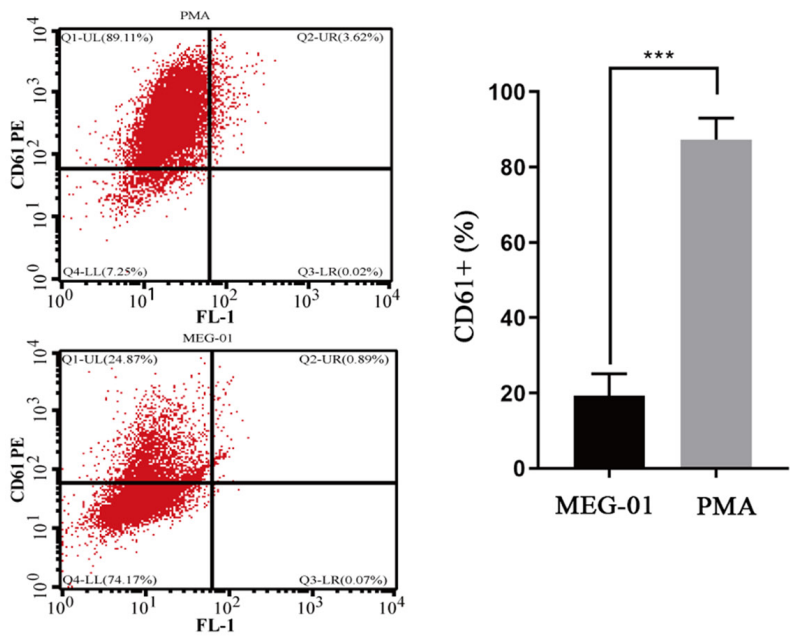

C

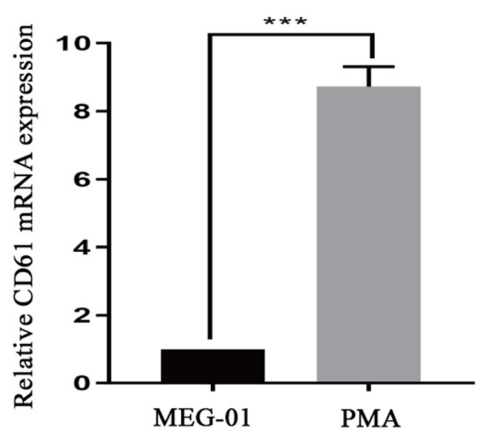

Figure 1. Induction of MEG-01 cell differentiation to platelets using PMA in vitro. MEG-01 cells were treated with PMA for $72 \mathrm{~h}$.(A) Cell morphological observations and Wright-Giemsa staining (magnification, x400). (B) CD61 cell surface expression was assessed via flow cytometry. (C) CD61 mRNA expression levels were determined via reverse transcription-quantitative PCR. ${ }^{* * *} \mathrm{P}<0.01$. PMA, phorbol-12-myristate-13-acetate; PE, phycoerythrin.

in vitro and suspended in RPMI-1640 supplemented with $10 \%$ FBS in the present study. Previous studies have reported that MEG-01 cells were successfully induced to differentiate into platelet-forming megakaryocytes following treatment with 15 ng/ml PMA for 72 h $(23,24)$. Morphological observations and Wright-Giemsa staining demonstrated that cells were larger in size with irregular edges and coarse granular chromatin structures, and were tightly arranged with unclear nucleoli prior to induction (Fig. 1A). After induction, cavitation occurred and pseudopods were generated. Following induction, the flow cytometry results demonstrated that CD61 expression on MEG-01 cells was significantly increased compared with untreated MEG-01 cells ( $\mathrm{P}<0.05$; Fig. 1B). Similarly, the RT-qPCR results also demonstrated that CD61 mRNA expression levels were significantly upregulated following induction compared with untreated MEG-01 cells $(\mathrm{P}<0.05$; Fig. 1C).

Effect of CCCP on platelet activity. The effect of CCCP on platelet activity was analyzed by performing CCK-8 assays. Compared with the control group (100\%), the survival rates of megakaryocytes/platelets following treatment with 10, 20, 50,70 or $100 \mu \mathrm{M}$ CCCP for $3 \mathrm{~h}$ were 77.57, 59.42, 48.13, 43.43 or $23.25 \%$, respectively (Fig. 2A). The results indicated that CCCP treatment decreased cell survival rates in a concentration-dependent manner. In addition, the expression levels of the autophagy-related protein, LC3-II/LC3-I, were significantly upregulated by CCCP treatment $(\geq 50 \mu \mathrm{M})$ compared with the $\mathrm{NC}$ group (Fig. 2B). Among the CCCP treatment groups, the highest expression levels of LC3-II/I were observed following treatment with $50 \mu \mathrm{M}$ CCCP for $3 \mathrm{~h}$. Therefore, treatment with $50 \mu \mathrm{M}$ CCCP for $3 \mathrm{~h}$ was selected as the optimal condition for the induction of mitochondrial autophagy in platelets.

PHB2 expression levels in platelets during mitophagy. Compared with the NC group, PHB2 protein expression levels were significantly upregulated by CCCP $(\geq 50 \mu \mathrm{M})$ treatment for $3 \mathrm{~h}$ in a dose-dependent manner in platelets (Fig. 3A). Following treatment with $50 \mu \mathrm{M}$ CCCP for $0,1,3,6,9$ or $12 \mathrm{~h}, \mathrm{PHB} 2$ protein expression levels were notably increased in a time-dependent manner in platelets compared with the NC group (Fig. 3B).

Effect of PHB2 knockdown on platelet mitophagy. Under the optimal established LV transfection conditions, the interfering fragments that effectively silenced PHB2 were screened. Platelets were divided into the following four groups: i) LV-PHB2-RNAi (57874-1); ii) LV-PHB2-RNAi (57875-1); iii) LV-PHB2-RNAi (57876-2); and iv) NC-RNAi (CON077). At $72 \mathrm{~h}$ post-transduction, transduction efficiency was assessed via RT-qPCR and western blotting. Subsequently, mRNA and protein were extracted from platelets in each group to measure PHB2 expression levels. PHB2 mRNA expression levels in the LV-PHB2-RNAi (57875-1) group were significantly downregulated compared with the NC-RNAi (CON077) group $(\mathrm{P}<0.05$; Fig. 4A). Similarly, PHB2 protein expression levels were also significantly downregulated in the LV-PHB2-RNAi (57875-1) group compared with the NC-RNAi (CON077) group ( $\mathrm{P}<0.05$ : Fig. 4B). PHB2 mRNA and protein expression levels among the other groups were not significantly different (Fig. 4). Therefore, LV-PHB2-RNAi (57875-1) was identified as the most effective interfering fragment of PHB2.

Effect of PHB2 expression on platelet mitophagy. Following the successful screening of the interference fragment of PHB2, cells were divided into the following groups: i) control (Con); ii) small interfering RNA (sh)-NC; and iii) sh-PHB2. Following successful lentiviral transduction, MEG-01 cells in each group were treated with PMA to induce the maturation and differentiation of the cells, and then treated with 0 or $50 \mu \mathrm{M} \mathrm{CCCP}$ for 
A

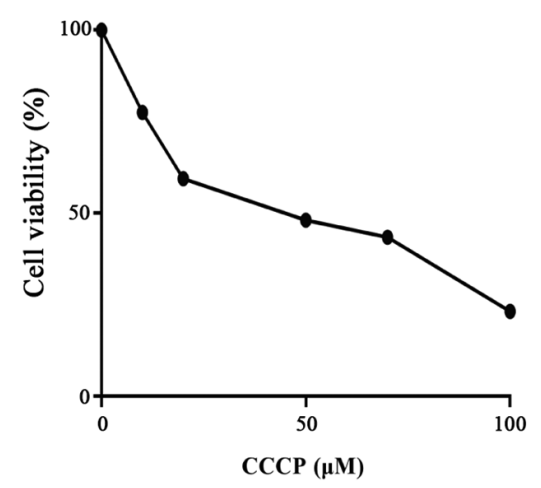

B

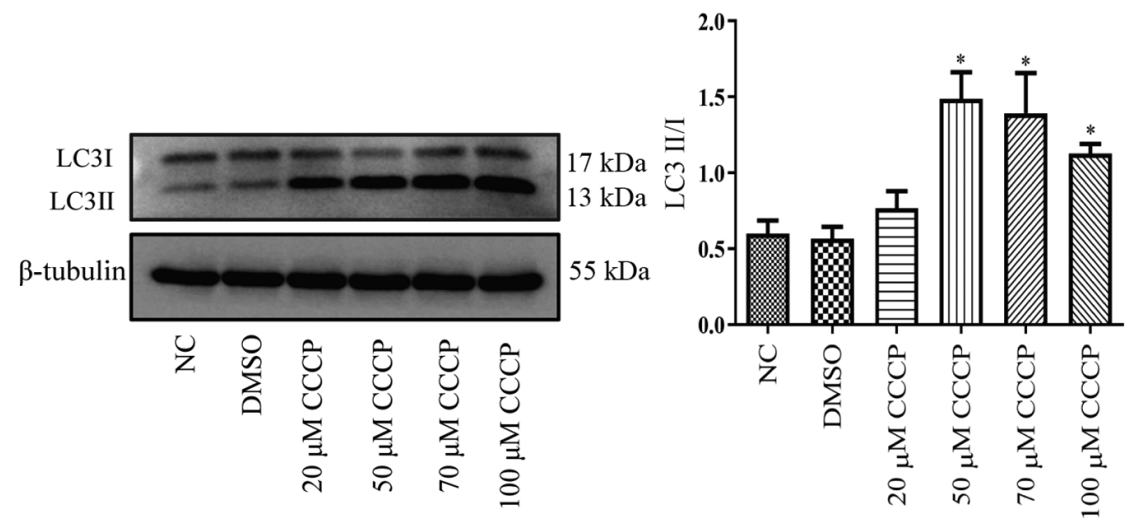

Figure 2. Effect of CCCP on platelet viability and LC3 expression. Following treatment with different concentrations of CCCP for $3 \mathrm{~h}$, (A) platelet cell viability and (B) LC3 protein expression levels were assessed. "P<0.05 vs. NC. CCCP, chlorophenylhydrazone; LC3, microtubule associated protein 1 light chain $3 \alpha$; $\mathrm{NC}$, negative control.

A

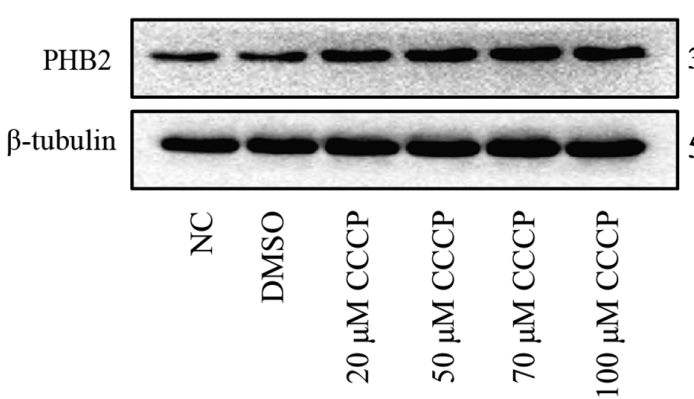

B

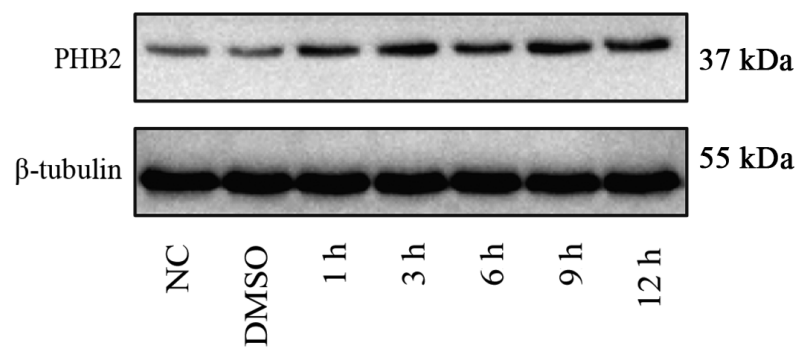

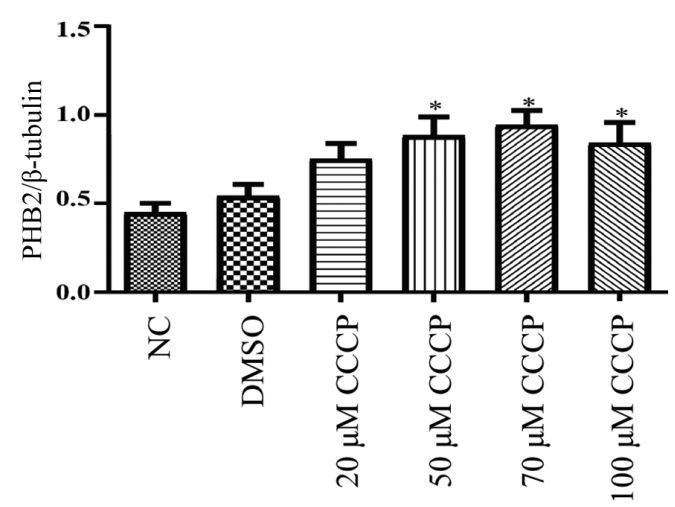

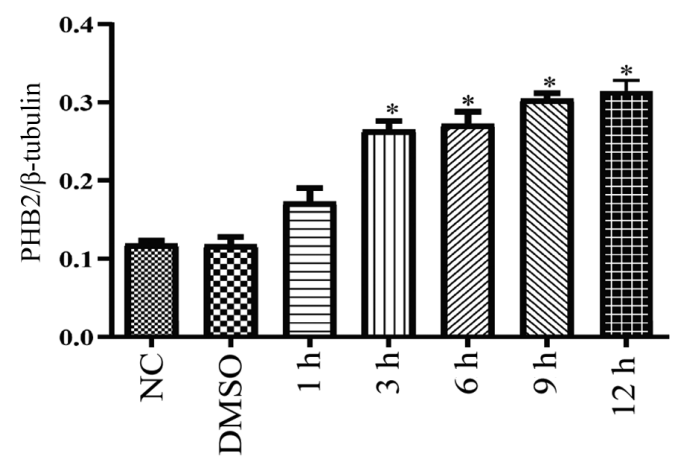

Figure 3. Effect of CCCP on PHB2 expression levels. (A) Following treatment with different concentrations of CCCP for 3 h, PHB2 protein expression levels were measured via western blotting. (B) Following treatment with $50 \mu \mathrm{M} \mathrm{CCCP}$ for 1,3,6,9 or $12 \mathrm{~h}, \mathrm{PHB} 2$ protein expression levels were measured via western blotting. PHB2 protein expression levels were upregulated in a time-dependent manner. " $\mathrm{P}<0.05$ vs. NC. CCCP, chlorophenylhydrazone; PHB2, prohibitin 2; $\mathrm{NC}$, negative control.

$3 \mathrm{~h}$. The western blotting results indicated that the expression levels of the autophagy-associated protein, LC3-II/LC3-I, were significantly upregulated in the sh-NC $+50 \mu \mathrm{M} \mathrm{CCCP}$ group compared with the $\mathrm{NC}+0 \mu \mathrm{M}$ CCCP group $(\mathrm{P}<0.01$; Fig. $5 \mathrm{~A})$. Furthermore, the LC3-II/LC3-I ratio was also significantly upregulated in the sh-PHB2 $+50 \mu \mathrm{M}$ CCCP group compared with the $\mathrm{NC}+0 \mu \mathrm{M} \mathrm{CCCP}$ group $(\mathrm{P}<0.001)$. The LC3-II/LC3-I ratio was significantly higher in the sh-PHB $2+50 \mu \mathrm{M} \mathrm{CCCP}$ group compared with the sh-NC $+50 \mu \mathrm{M}$ CCCP group $(\mathrm{P}<0.05)$, which suggested that $\mathrm{PHB} 2$ knockdown may increase the level of autophagy in platelets. In addition, the $\Delta \Psi_{\mathrm{m}}$ was analyzed via flow cytometry. The results revealed that the $\Delta \Psi_{\mathrm{m}}$ was significantly decreased in the sh-NC $+50 \mu \mathrm{M} \mathrm{CCCP}$ group compared with the $\mathrm{NC}+0 \mu \mathrm{M}$ CCCP group $(\mathrm{P}<0.01$; Fig. 5B). Similarly, the $\Delta \Psi_{\mathrm{m}}$ level in the sh-PHB2 $+50 \mu \mathrm{M}$ CCCP group was also significantly decreased compared with the $\mathrm{NC}+0 \mu \mathrm{M}$ CCCP group $(\mathrm{P}<0.001)$. Moreover, the $\Delta \Psi_{\mathrm{m}}$ level was significantly reduced in the sh-PHB2 $+50 \mu \mathrm{M}$ $\mathrm{CCCP}$ group compared with the sh- $\mathrm{NC}+50 \mu \mathrm{M} \mathrm{CCCP}$ group $(\mathrm{P}<0.01)$. 
A

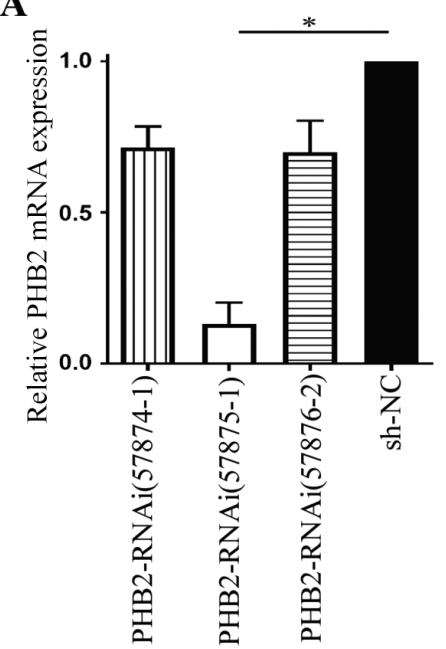

B
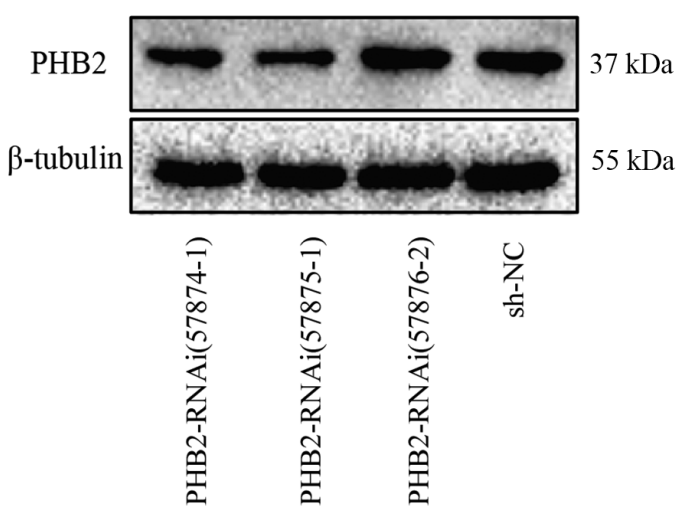

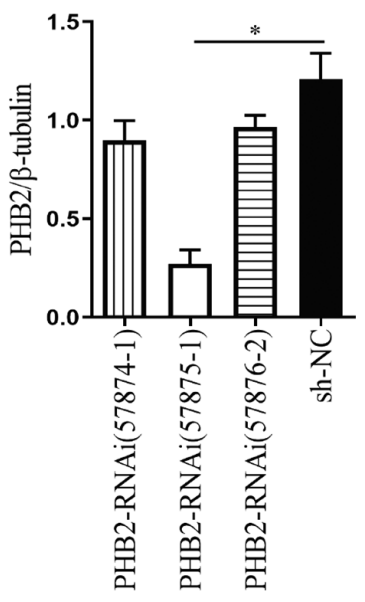

Figure 4. PHB2 knockdown in platelets. PHB2 (A) mRNA and (B) protein expression levels in platelets were analyzed via reverse transcription-quantitative PCR and western blotting, respectively. ${ }^{\mathrm{P}}<0.05$. PHB2, prohibitin 2; NC, negative control; RNAi, interference RNA.

A

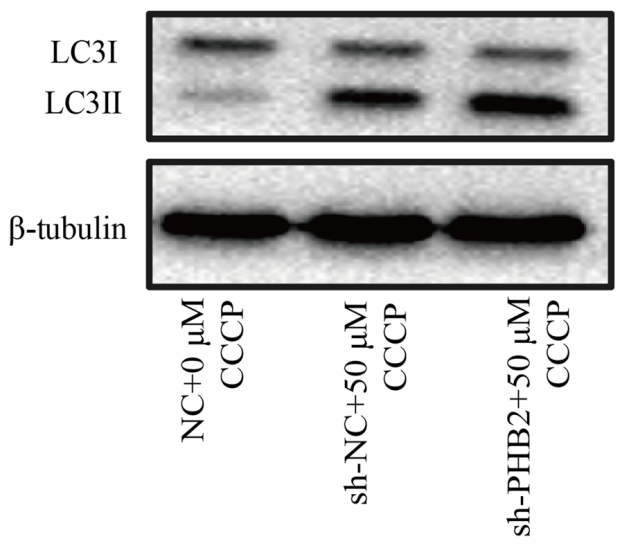

$13 \mathrm{kDa}$

$17 \mathrm{kDa}$

$55 \mathrm{kDa}$

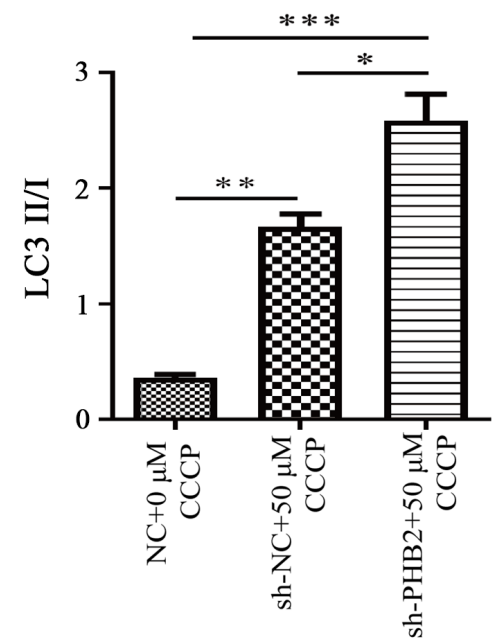

B
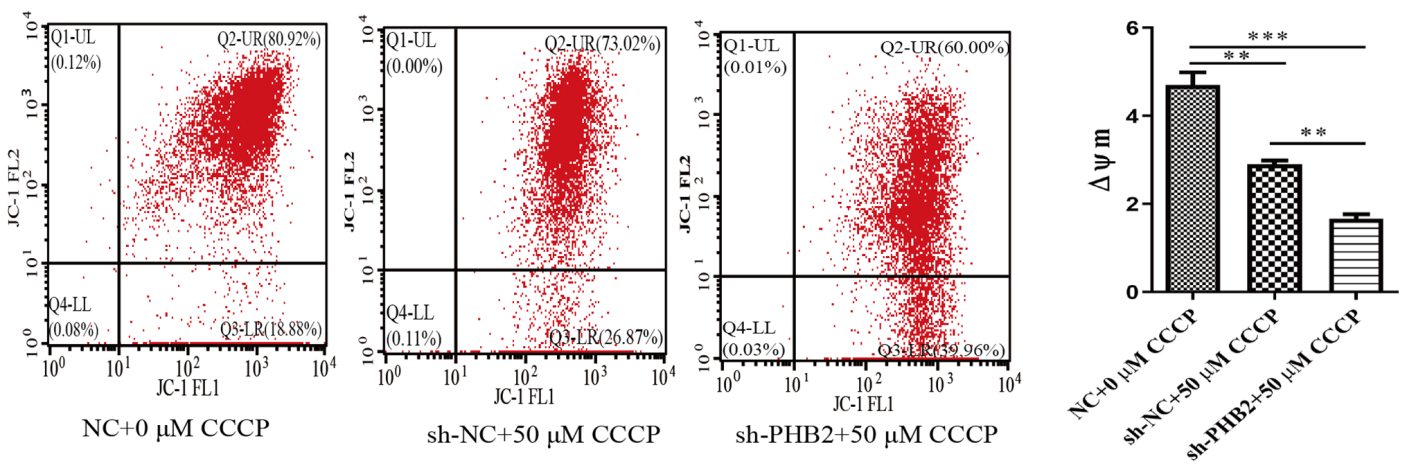

Figure 5. Effect of PHB2 on platelet mitophagy. (A) LC3 protein expression levels were measured via western blotting. (B) Flow cytometry was used to determine the $\Delta \Psi_{\mathrm{m}}$ in platelets. ${ }^{*} \mathrm{P}<0.05,{ }^{* *} \mathrm{P}<0.01$ and ${ }^{* * *} \mathrm{P}<0.001 . \Delta \Psi_{\mathrm{m}}$, mitochondrial membrane potential; NC, negative control; sh, short hairpin RNA; CCCP, chlorophenylhydrazone.

Transcriptome sequencing analysis of platelet activation following PHB2 knockdown. After mRNA-seq, 2.4 million clean reads were obtained, of which $85.96 \%$ were aligned to the exon region, whereas the remaining reads were distributed in the intronic and intergenic regions. Compared with the sh-NC group, 323 differentially expressed transcripts in the sh-PHB2 group were screened (Fig. 6A). A total of 12,554 DEGs were screened from the NC and sh-PHB2 groups by differential gene screening (Fig. 6B). KEGG signaling pathway enrichment analysis of the DEGs revealed that 
$\mathbf{A}$

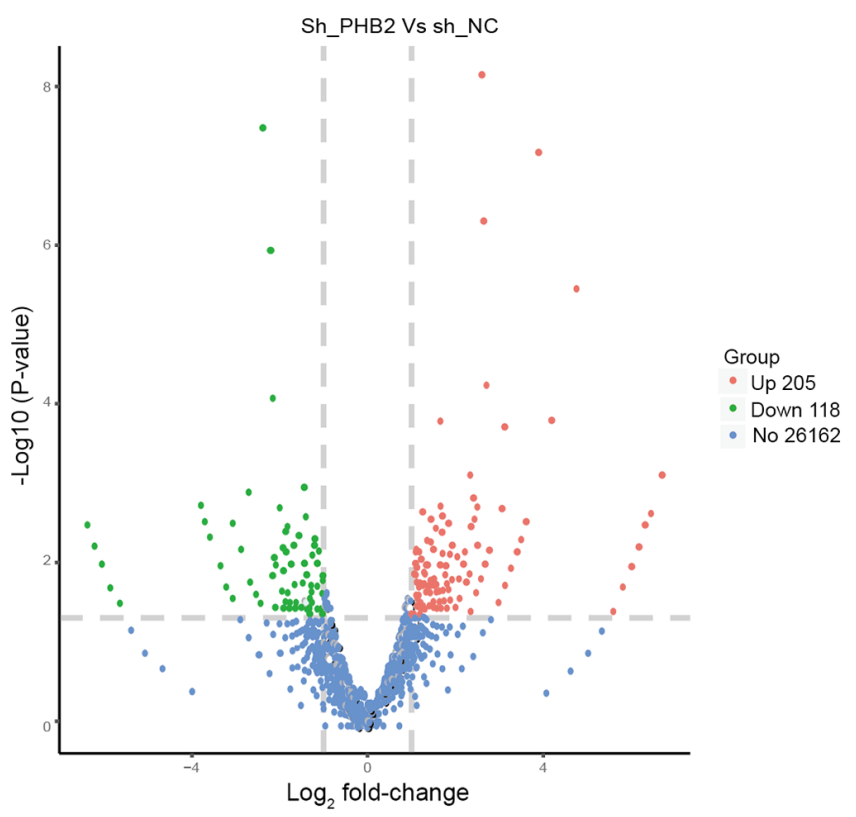

B

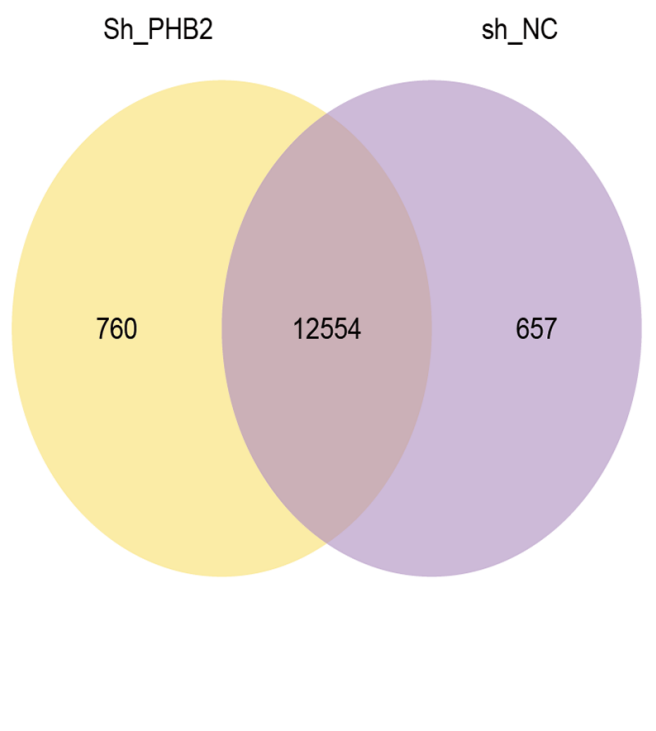

Figure 6. Transcriptome sequencing analysis of platelet activation following PHB2 knockdown. (A) Transcriptome of the screened differentially expressed genes. (B) Venn diagram of the differentially expressed genes between the NC and shPHB2 groups. NC, negative control; sh, short hairpin RNA; PHB2, prohibitin 2 .

Table II. Analysis of platelet-associated activation gene signaling pathways.

\begin{tabular}{lcrcc}
\hline Gene & shPHB2 & \multicolumn{1}{c}{ NC } & log2-FoldChange & P-value \\
\hline COL1A2 & 13.78 & 3.05 & 2.13 & $<0.05$ \\
TLN2 & 61.04 & 27.42 & 1.15 & $<0.05$ \\
NOS3 & 51.19 & 23.36 & 1.13 & $<0.05$ \\
COL3A1 & 34.46 & 14.22 & 1.27 & $<0.05$ \\
\hline
\end{tabular}

COL1A2, collagen type 1 a 2 chain; TLN2, talin 2; NOS3, nitric oxide synthase 3 ; COL $3 \mathrm{~A} 1$, collagen type III $\alpha 1$ chain; sh, short hairpin RNA; PHB2; NC, negative control.

285 DEGs were enriched in 183 KEGG pathways. Statistical analysis identified 20 significant KEGG signaling pathways, including 'Focal adhesion', 'ECM-receptor interaction' and 'Apelin signaling pathway' (adjusted $\mathrm{P}<0.05$; Fig. 7). Among these, genes that were associated with the platelet activation pathway included collagen type $1 \alpha 2$ chain (COL1A2), talin 2 (TLN2), nitric oxide synthase 3 (NOS3) and collagen type III $\alpha 1$ chain (COL3A1) (Table II).

\section{Discussion}

Previous studies have reported that the inner mitochondrial membrane protein PHB2 is a key mitophagy receptor $(25,26)$. Mitophagy is a specific selection process regulated by various factors, including ischemia, other cardiac stress or heart disease conditions, that regulates the number of mitochondria that adapt to the cell survival environment (27). To the best of our knowledge, the regulation of PHB2 in the process of platelet mitophagy has not been previously reported; thus, for the first time, transcriptomics sequencing in the present study discovered PHB2-mediated regulation of downstream platelet activation related genes. However, the precise mechanisms underlying mitophagy are not completely understood.

PHB2 is a highly conserved inner mitochondrial membrane protein that has been reported to regulate mitochondrial assembly and function due to its unique localization on the mitochondria membrane $(5,28)$. Wei et al $(25)$ previously reported that mitochondrial receptors were involved in the mitochondrial degradation of PHB2, and mitophagy was inhibited following PHB2 knockdown, which was a similar effect to that observed following autophagy related 7 (ATG7) knockdown. In addition, mitochondrial respiratory inhibitor-induced mitophagy significantly upregulated PHB2 expression levels, which were subsequently blocked following transfection with si-ATG7, indicating that PHB2 may serve as a marker of mitophagy (29). Xiao et al (26) also reported that PHB2 was required for cholestasis-induced mitophagy, whereby PHB2 transferred LC3 to damaged mitochondria by interacting with sequestosome 1 and LC3, which subsequently maintained homeostasis. The aforementioned results suggested that PHB2 may also serve an important role in the development of platelet mitophagy.

The present study aimed to investigate the role of PHB2 in the induction of a differentiated platelet model. CCCP was used as an inducer of mitochondrial autophagy, as it has been reported to directly act on mitochondria, uncouple mitochondria and damage the membrane potential (15). Previous literature indicated that $\mathrm{CCCP}$ should be used at a concentration range of $10-100 \mu \mathrm{M}$; therefore, the present study analyzed the most effective concentration by performing CCK- 8 assays and western blotting. In the present study, $50 \mu \mathrm{M}$ CCCP was used to induce platelet mitophagy. As platelets derived from 


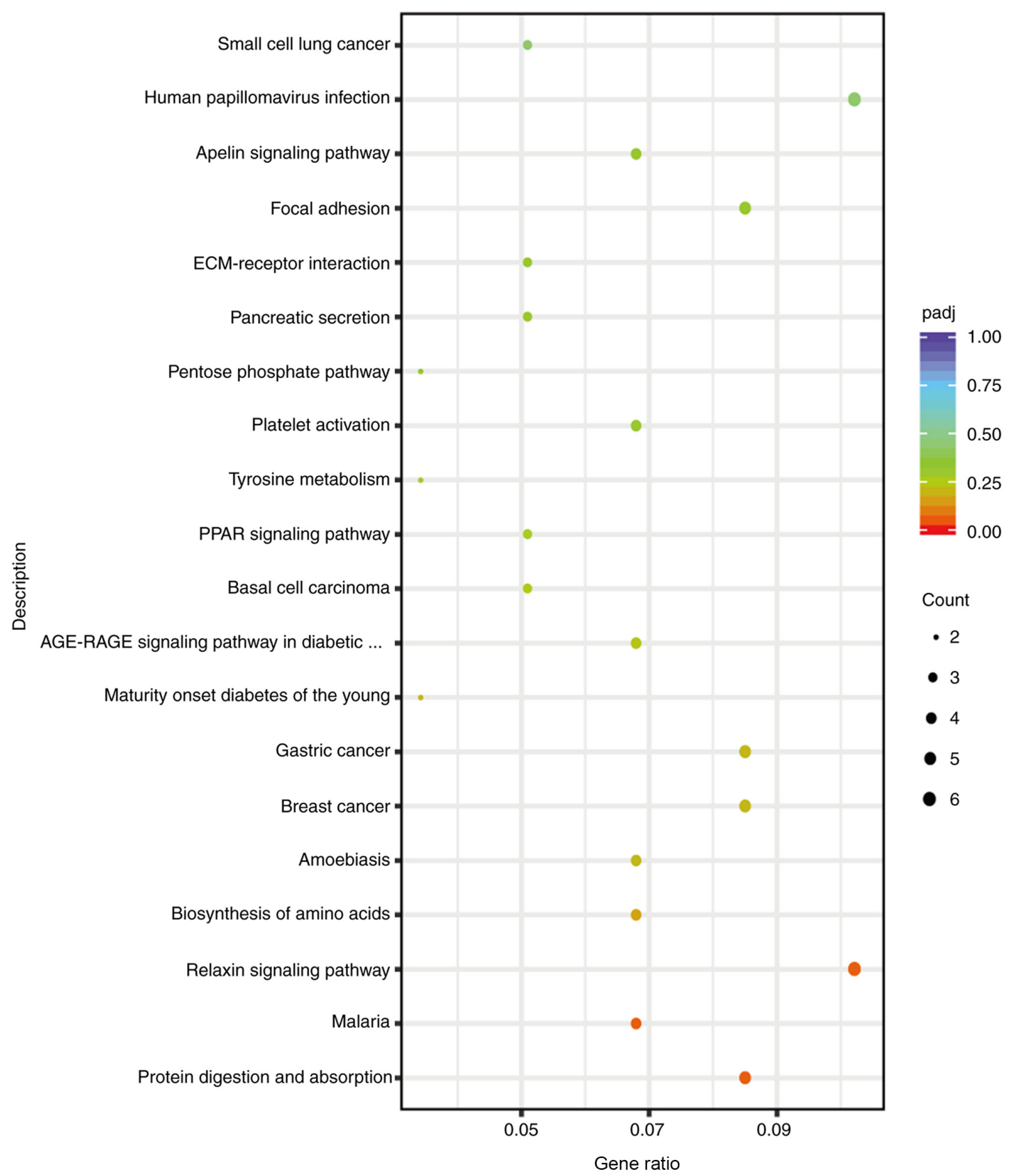

Figure 7. Analysis of activated genes affected by PHB2 in platelet KEGG enrichment. Kyoto Encyclopedia of Genes and Genomes signaling pathway enrichment analysis of the effect of differentially expressed genes on the platelet activation pathway.

the differentiation of the MEG-01 cell line were used in the present study, the platelets may have been more tolerable and relatively insensitive to stimulation compared with platelets directly isolated from humans or animals. The aforementioned hypothesis may provide an explanation for the higher concentration of CCCP used in the present study compared with previous studies $(16,17)$. Following treatment with 70 or $100 \mu \mathrm{M} \mathrm{CCCP}$, platelet viability was notably inhibited compared with the $\mathrm{NC}$ group in the present study. Autophagy is a double-edged sword that displays a protective effect at an appropriate levels, but the level of autophagy can be increased. At high concentrations, excessive autophagy activation occurs, which presents as decreased levels of autophagy protein molecule LC3II/I (30). The results of the present study demonstrated that PHB2 was involved in platelet mitophagy, and its expression levels were altered according to the concentration and duration of CCCP treatment. Moreover, the results indicated that PHB2 protein expression levels were upregulated in a dose- and time-dependent manner. Therefore, the present study suggested that PHB2 may serve as a mitophagy receptor, maintaining the stability of the mitochondrial structure in platelets.

Since PHB2 is widely expressed in biological cells, distinguishing its role in mitochondria compared with other organelles or cytoplasm is important. Lee et al (15) demonstrated that CCCP could induce mitophagy in individual platelets. Therefore, $\mathrm{CCCP}$, a proton carrier $\left(\mathrm{H}^{+}\right.$ionophore), is a powerful uncoupling agent for mitochondrial oxidative phosphorylation. CCCP enhances the permeability of the mitochondrial inner membrane to $\mathrm{H}^{+}$, resulting in the loss of 
the membrane potential on both sides of the mitochondrial inner membrane, which ultimately leads to mitophagy $(15,31)$. Although CCCP-induced PHB2-mediated mitophagy cannot directly reflect the precise positioning and function of PHB2 on the mitochondria, it can be partially speculated that it is caused by the changes of PHB2 following CCCP-induced mitophagy.

PHB2 has been previously reported to serve an important role in platelets. For example, PHB2 participated in Prader Willi/Angelman region RNA 1-mediated platelet aggregation, and regulated thrombocyte activation and related thrombin-induced signaling pathways (32). Therefore, it was hypothesized that PHB2 may serve an important role in regulating mitochondrial function and platelet activation. Previous studies have reported the contribution of several mitochondrial functions in platelet activation, including increased mitochondrial reactive oxygen species (mtROS) generation (33-35), a decrease in the $\Delta \Psi_{m}(36)$ and the induction of mitochondrial dysfunction (31). In type II diabetes mellitus, ROS production, an increased mitochondrial mass, higher $\Delta \Psi m$ and elevated levels of mitochondrial respiration were reported be associated with platelet activation $(37,38)$. Therefore, it was hypothesized that similar processes, including increased mtROS generation, a higher $\Delta \Psi m$ and an elevated mitochondrial mass, may also occur in platelets during mitophagy. By using JC-1 as the main indicator for evaluating platelet mitochondrial function, the results of the present study revealed that the $\Delta \Psi m$ was significantly decreased in the sh-NC $+50 \mu \mathrm{M} \mathrm{CCCP}$ and sh-PHB2 $+50 \mu \mathrm{M} \mathrm{CCCP}$ groups compared with the $\mathrm{NC}+0 \mu \mathrm{M}$ CCCP group. $\Delta \Psi_{\mathrm{m}}$ refers to the difference in transmembrane potential produced by the different ion concentrations on both sides of the inner mitochondrial membrane, which is the driving force for ATP synthesis and release (36). Thus, the $\Delta \Psi m$ is a sensitive indicator for evaluating mitochondrial function. The results of the present study also demonstrated that PHB2 knockdown significantly decreased the $\Delta \Psi_{\mathrm{m}}$ in $50 \mu \mathrm{M}$ CCCP-treated cells, which also resulted in upregulation of the LC3-II/LC3-I ratio. A potential mechanism underlying PHB2 knockdown-mediated effects is that PHB2 served as a specific inner mitochondrial membrane receptor. As a result of autophagy inhibition due to PHB2 knockdown, damaged or redundant mitochondria were not removed in time and the stability of the structure and function of mitochondria was not maintained in vivo, leading to a decrease in the $\Delta \Psi_{\mathrm{m}}$ and the accumulation of the autophagy-related protein, LC3.

Zhang et al (39) reported for the first time that FUN14 domain containing 1 (FUNDC1)-mediated mitophagy in platelets was crucial for ischemic adaptation in response to hypoxia, which serves a crucial role in platelet activation. Furthermore, the aforementioned study also identified BCL2 interacting protein 3 like as a receptor that mediated platelet mitophagy by regulating the mitophagy flux, as a housekeeping process, to maintain mitochondrial quality in platelets (40). The aforementioned results suggested the existence of an association between receptor-mediated platelet mitophagy and platelet activation. Therefore, further investigation into PHB2-mediated platelet mitophagy may identify an important mechanism for the prevention and treatment of cardiovascular diseases. Increasing evidence has suggested that hypoxic preconditioning in the clinic reduces ischemia-reperfusion (I/R)-induced-heart injury $(41,42)$. Furthermore, hypoxic preconditioning was reported to induce mitophagy in platelets and aid with determining the outcome of I/R injury (43). Of particular interest, FUNDC1-mediated mitophagy in cardiomyocytes and platelets was identified as a major mechanism underlying cardioprotection against I/R-induced heart injury. Under normal physiological conditions, damaged toxic platelet mitochondria are removed to maintain mitochondrial quality and platelet activation. Platelets adhere to the site of injury to reduce blood oxygen levels, which promotes myocardial infarction. Subsequently, the occlusion of the blood vessels creates a hypoxic environment that triggers platelet mitophagy to lower platelet activity, thereby preventing I/R damage from worsening (44). It was hypothesized that PHB2 may also be involved in I/R-induced heart injury; however, the underlying mechanism requires further investigation.

In the present study, the transcriptome sequencing analysis revealed that $\mathrm{PHB} 2$ knockdown altered the expression of platelet-associated activation genes, including COL1A2, TLN2, NOS3 and COL3A1. Type I and III collagen encoded by the COL1A 2 and COL3A1 genes, respectively, were previously reported to serve an important role in activating platelets, forming thrombi and maintaining the elasticity of the arterial wall (45-47). Another previous study reported that NOS3 was an endothelial-induced NOS that was involved in endothelial NOS signaling transduction and regulation of nitric oxide (NO) in vivo $(48,49)$. The synthesis of NO not only altered endothelial function (50), but also affected platelet activity. For example, the release of NO inhibited platelet aggregation and adhesion functions to a certain degree (51). However, the role of TLN2 in the context of platelet activation has not been reported and requires further investigation. The present study aimed to investigate platelet activation and mitophagy. However, a key limitation of the present study was that other platelet functions were not investigated.

In conclusion, the present study demonstrated that PHB2, as an inner mitochondrial membrane receptor, may be involved in platelet mitophagy and inhibit platelet activation by downregulating the expression of platelet activation genes. However, the related functions and mechanisms underlying PHB2 require further investigation. It was hypothesized that PHB2 may influence the activation of platelets by altering the function of mitochondria; therefore, $\mathrm{PHB} 2$ may serve as a novel therapeutic target for thrombosis-related diseases due to its unique localization on the mitochondrial membrane.

\section{Acknowledgements}

Not applicable.

\section{Funding}

The present study was supported by the National Natural Science Foundation of China (grant nos. 81960081 and 81660063) and the Natural Science Foundation of Jiangxi (grant no. 20171BAB205041).

\section{Availability of data and materials}

The datasets used and/or analyzed during the current study are available from the corresponding author on reasonable 
request. The sequencing data were submitted to Sequence Read Archive (accession no. PRJNA692313; www.ncbi.nlm. nih.gov/bioproject/PRJNA692313).

\section{Authors' contributions}

LLH and KZ conceived and designed the study, analyzed the data and wrote the manuscript. LLH, KZ, YC, LJW, JC, XYX, LW and XSC performed the experiments, and analyzed and interpreted the data. LLH and KZ confirm the authenticity of all the raw data. QZX and RQY designed the study and critically revised the manuscript. RQY guaranteed the work, had full access to all the data in the study and takes responsibility for the integrity of the data and the accuracy of the data analysis. All authors read and approved the final version of the manuscript.

\section{Ethics approval and consent to participate}

Not applicable.

\section{Patient consent for publication}

Not applicable.

\section{Competing interests}

The authors declare that they have no competing interests.

\section{References}

1. Snedden WA and Fromm H: Characterization of the plant homologue of prohibitin, a gene associated with antiproliferative activity in mammalian cells. Plant Mol Biol 33: 753-756, 1997.

2. Loyer X, Potteaux S, Vion AC, Guerin CL, Boulkroun S, Rautou PE, Ramkhelawon B, Esposito B, Dalloz M, Paul JL, et al: Inhibition of microRNA-92a prevents endothelial dysfunction and atherosclerosis in mice. Circ Res 114: 434-443, 2014.

3. Merkwirth $\mathrm{C}$ and Langer T: Prohibitin function within mitochondria: Essential roles for cell proliferation and cristae morphogenesis. Biochim Biophys Acta 1793: 27-32, 2009.

4. Osman C, Merkwirth $C$ and Langer T: Prohibitins and the functional compartmentalization of mitochondrial membranes. J Cell Sci 122: 3823-3830, 2009.

5. Artal-Sanz M and Tavernarakis N: Opposing function of mitochondrial prohibitin in aging. Aging (Albany NY) 2: 1004-1011, 2010.

6. Guo X, Wu J, Du J, Ran J and Xu J: Platelets of type 2 diabetic patients are characterized by high ATP content and low mitochondrial membrane potential. Platelets 20: 588-593, 2009.

7. Avila C, Huang RJ, Stevens MV, Aponte AM, Tripodi D, Kim KY and Sack MN: Platelet mitochondrial dysfunction is evident in type 2 diabetes in association with modifications of mitochondrial anti-oxidant stress proteins. Exp Clin Endocrinol Diabetes 120: 248-251, 2012

8. Fuentes E, Araya-Maturana R and Urra FA: Regulation of mitochondrial function as a promising target in platelet activation-related diseases. Free Radic Biol Med 136: 172-182, 2019.

9. Xue L, Fletcher GC and Tolkovsky AM: Mitochondria are selectively eliminated from eukaryotic cells after blockade of caspases during apoptosis. Curr Biol 11: 361-365, 2001.

10. Lemasters JJ: Selective mitochondrial autophagy, or mitophagy, as a targeted defense against oxidative stress, mitochondrial dysfunction, and aging. Rejuvenation Res 8: 3-5, 2005.

11. Bhatia-Kissova I and Camougrand N: Mitophagy: A process that adapts to the cell physiology. Int J Biochem Cell Biol 45: 30-33, 2013.

12. Pieczarka EM, Yamaguchi M, Wellman ML and Judith Radin M: Platelet vacuoles in a dog with severe nonregenerative anemia: Evidence of platelet autophagy. Vet Clin Pathol 43: 326-329, 2014.
13. Feng W, Chang C, Luo D, Su H, Yu S, Hua W, Chen Z, Hu H and Liu W: Dissection of autophagy in human platelets. Autophagy 10: 642-651, 2014.

14. Cao Y, Cai J, Zhang S, Yuan N, Li X, Fang Y, Song L, Shang M, Liu S, Zhao W, et al: Loss of autophagy leads to failure in megakaryopoiesis, megakaryocyte differentiation, and thrombopoiesis in mice. Exp Hematol 43: 488-494, 2015.

15. Lee SH, Du J, Stitham J, Atteya G, Lee S, Xiang Y, Wang D, Jin Y, Leslie KL, Spollett G, et al: Inducing mitophagy in diabetic platelets protects against severe oxidative stress. EMBO Mol Med 8: 779-795, 2016.

16. Griffith JM, Basting PJ, Bischof KM, Wrona EP, Kunka KS, Tancredi AC, Moore JP, Hyman MRL and Slonczewski JL: Experimental evolution of Escherichia coli K-12 in the presence of proton motive force (PMF) uncoupler carbonyl cyanide m-chlorophenylhydrazone selects for mutations affecting PMF-driven drug efflux pumps. Appl Environ Microbiol 85: e02792-e02718, 2019.

17. Rodriguez C, Simon V, Conget P and Vega IA: Both quiescent and proliferating cells circulate in the blood of the invasive apple snail Pomacea canaliculata. Fish Shellfish Immunol 107: 95-103, 2020.

18. Livak KJ and Schmittgen TD: Analysis of relative gene expression data using real-time quantitative PCR and the 2(-Delta Delta C(T)) method. Methods 25: 402-408, 2001.

19. Trapnell C, Williams BA, Pertea G, Mortazavi A, Kwan G, van Baren MJ, Salzberg SL, Wold BJ and Pachter L: Transcript assembly and quantification by RNA-Seq reveals unannotated transcripts and isoform switching during cell differentiation. Nat Biotechnol 28: 511-515, 2010.

20. Young MD, Wakefield MJ, Smyth GK and Oshlack A: Gene ontology analysis for RNA-seq: Accounting for selection bias. Genome Biol 11: R14, 2010.

21. Kanehisa M, Araki M, Goto S, Hattori M, Hirakawa M, Itoh M, Katayama T, Kawashima S, Okuda S, Tokimatsu T and Yamanishi Y: KEGG for linking genomes to life and the environment. Nucleic Acids Res 36: D480-D484, 2008.

22. Mao X, Cai T, Olyarchuk JG and Wei L: Automated genome annotation and pathway identification using the KEGG orthology (KO) as a controlled vocabulary. Bioinformatics 21: 3787-3793, 2005.

23. Ogura M, Morishima Y, Okumura M, Hotta T, Takamoto S, Ohno R, Hirabayashi N, Nagura H and Saito H: Functional and morphological differentiation induction of a human megakaryoblastic leukemia cell line (MEG-01s) by phorbol diesters. Blood 72: 49-60, 1988.

24. Takeuchi K, Satoh M, Kuno H, Yoshida T, Kondo H and Takeuchi M: Platelet-like particle formation in the human megakaryoblastic leukaemia cell lines, MEG-01 and MEG-01s. Br J Haematol 100: 436-444, 1998.

25. Wei Y, Chiang WC, Sumpter R Jr, Mishra P and Levine B: Prohibitin 2 is an inner mitochondrial membrane mitophagy receptor. Cell 168: 224-238.e10, 2017.

26. Xiao Y, Zhou Y, Lu Y, Zhou K and Cai W: PHB2 interacts with LC3 and SQSTM1 is required for bile acids-induced mitophagy in cholestatic liver. Cell Death Dis 9: 160, 2018.

27. Galluzzi L, Bravo-San Pedro JM and Kroemer G: Mitophagy: Permitted by prohibitin. Curr Biol 27: R73-R76, 2017.

28. Bavelloni A, Piazzi M, Raffini M, Faenza I and Blalock WL: Prohibitin 2: At a communications crossroads. IUBMB Life 67: 239-254, 2015.

29. Lahiri V and Klionsky DJ: PHB2/prohibitin 2: An inner membrane mitophagy receptor. Cell Res 27: 311-312, 2017.

30. Schiattarella GG and Hill JA: Therapeutic targeting of autophagy in cardiovascular disease. J Mol Cell Cardiol 95: 86-93, 2016.

31. Wang Y, Nartiss Y, Steipe B, McQuibban GA and Kim PK: ROS-induced mitochondrial depolarization initiates PARK2/PARKIN-dependent mitochondrial degradation by autophagy. Autophagy 8: 1462-1476, 2012.

32. Zhang Y, Wang Y, Xiang Y, Lee W and Zhang Y: Prohibitins are involved in protease-activated receptor 1-mediated platelet aggregation. J Thromb Haemost 10: 411-418, 2012.

33. Lopez JJ, Salido GM, Gómez-Arteta E, Rosado JA and Pariente JA: Thrombin induces apoptotic events through the generation of reactive oxygen species in human platelets. J Thromb Haemost 5: 1283-1291, 2007.

34. Yamagishi SI, Edelstein D, Du XL and Brownlee M: Hyperglycemia potentiates collagen-induced platelet activation through mitochondrial superoxide overproduction. Diabetes 50: 1491-1494, 2001 
35. Choo HJ, Saafir TB, Mkumba L, Wagner MB and Jobe SM: Mitochondrial calcium and reactive oxygen species regulate agonist-initiated platelet phosphatidylserine exposure. Arterioscler Thromb Vasc Biol 32: 2946-2955, 2012.

36. Jobe SM, Wilson KM, Leo L, Raimondi A, Molkentin JD, Lentz SR and Di Paola J: Critical role for the mitochondrial permeability transition pore and cyclophilin $\mathrm{D}$ in platelet activation and thrombosis. Blood 111: 1257-1265, 2008.

37. Siewiera K, Kassassir H, Talar M, Wieteska L and Watala C: Higher mitochondrial potential and elevated mitochondrial respiration are associated with excessive activation of blood platelets in diabetic rats. Life Sci 148: 293-304, 2016.

38. Wu F, Liu Y, Luo L, Lu Y, Yew D, Xu J and Guo K: Platelet mitochondrial dysfunction of DM rats and DM patients. Int J Clin Exp Med 8: 6937-6946, 2015.

39. Zhang W, Ren H, Xu C, Zhu C, Wu H, Liu D, Wang J, Liu L, $\mathrm{Li} \mathrm{W}, \mathrm{Ma} \mathrm{Q}$, et al: Hypoxic mitophagy regulates mitochondrial quality and platelet activation and determines severity of I/R heart injury. Elife 5: e21407, 2016.

40. Zhang W, Ma Q, Siraj S, Ney PA, Liu J, Liao X, Yuan Y, Li W, Liu L and Chen Q: Nix-mediated mitophagy regulates platelet activation and life span. Blood Adv 3: 2342-2354, 2019.

41. Eitel I, Stiermaier T, Rommel KP, Fuernau G, Sandri M, Mangner N, Linke A, Erbs S, Lurz P, Boudriot E, et al: Cardioprotection by combined intrahospital remote ischaemic perconditioning and postconditioning in ST-elevation myocardial infarction: The randomized LIPSIA CONDITIONING trial. Eur Heart J 36: 3049-3057, 2015

42. Hausenloy DJ, Barrabes JA, Bøtker HE, Davidson SM, Di Lisa F, Downey J, Engstrom T, Ferdinandy P, Carbrera-Fuentes HA, Heusch G, et al: Ischaemic conditioning and targeting reperfusion injury: A 30 year voyage of discovery. Basic Res Cardiol 111: $70,2016$.

43. Zhang W, Siraj S, Zhang R and Chen Q: Mitophagy receptor FUNDC1 regulates mitochondrial homeostasis and protects the heart from I/R injury. Autophagy 13: 1080-1081, 2017.
44. Zhang W, Chen C, Wang J, Liu L, He Y and Chen Q: Mitophagy in cardiomyocytes and in platelets: A major mechanism of cardioprotection against ischemia/reperfusion injury. Physiology (Bethesda) 33: 86-98, 2018.

45. Penz S, Reininger AJ, Brandl R, Goyal P, Rabie T, Bernlochner I, Bernlochner I, Rother E, Goetz C, Engelmann B, et al: Human atheromatous plaques stimulate thrombus formation by activating platelet glycoprotein VI. FASEB J 19: 898-909, 2005.

46. Lv W, Lin Y, Song W, Sun K, Yu H, Zhang Y, Zhang C, Li L, Suo M, Hui R and Chen J: Variants of COL3A1 are associated with the risk of stroke recurrence and prognosis in the Chinese population: A prospective study. J Mol Neurosci 53: 196-203, 2014.

47. Buxhofer-Ausch V, Steurer M, Sormann S, Schloegl E, Schimetta W, Gisslinger B, Ruckser R, Gastl G and Gisslinger H: Influence of platelet and white blood cell counts on major thrombosis-analysis from a patient registry in essential thrombocythemia. Eur J Haematol 97: 511-516, 2016.

48. Fleming I: Molecular mechanisms underlying the activation of eNOS. Pflugers Arch 459: 793-806, 2010.

49. Xia N, Forstermann U and Li H: Resveratrol and endothelial nitric oxide. Molecules 19: 16102-16121, 2014.

50. Khan M, Meduru S, Gogna R, Madan E, Citro L, Kuppusamy ML, Sayyid M, Mostafa M, Hamlin RL and Kuppusamy P: Oxygen cycling in conjunction with stem cell transplantation induces NOS3 expression leading to attenuation of fibrosis and improved cardiac function. Cardiovasc Res 93: 89-99, 2012.

51. Bharath LP, Mueller R, Li Y, Ruan T, Kunz D, Goodrich R, Mills T, Deeter L, Sargsyan A, Babu PVA, et al: Impairment of autophagy in endothelial cells prevents shear-stress-induced increases in nitric oxide bioavailability. Can J Physiol Pharmacol 92: 605-612, 2014.

This work is licensed under a Creative Commons

Attribution-NonCommercial-NoDerivatives 4.0 International (CC BY-NC-ND 4.0) License. 